\title{
Stroke criptogénico y foramen oval permeable
}

\author{
Cryptogenic stroke and patent foramen ovale
}

Horacio José Faella', Germán Henestrosa²

\section{RESUMEN}

Se ha establecido una fuerte asociación entre foramen oval permeable (FOP) y stroke criptogénico. El mecanismo sería una embolia paradójica en la cual un trombo venoso pasa a través del FOP hacia la circulación sistémica y provoca una embolia cerebral. El FOP es relativamente común en la población general, pero su prevalencia es mayor en los pacientes con stroke criptogénico.

Existe además una relación entre el FOP y la enfermedad descompresiva neurológica de los buceadores, el síndrome platipnea-ortodeoxia y las migrañas.

Hay formas de tratamiento: médico (con antiagregantes o anticoagulantes) y el cierre del FOP (quirúrgico o percutáneo).

Para pacientes con historia de stroke criptogénico y FOP, el estudio RESPECT provee evidencia que el cierre con oclusor PFO Amplatzer reduce el riesgo de recurrencia entre un 46,6 y $72,7 \%$ sobre el tratamiento médico con anticoagulantes o antiagregantes.

Palabras claves: stroke, foramen oval permeable, RESPECT.

\begin{abstract}
There have been established a strong association between the presence of patent foramen ovale (PFO) and documented stroke of unknown cause, commonly referred to as cryptogenic stroke. The mechanism involved is presumed to be a paradoxical embolism from a venous thrombus that travels via the PFO to the systemic circulation. $\mathrm{PFO}$ is relatively common in the general population, but its prevalence is higher in patients with cryptogenic stroke. There are four major forms of treatment: 1) medical therapy with antiplatelet agents, 2) medical therapy with anticoagulants, 3) surgical closure, and 4) percutaneous device closure. The PFO in Cryptogenic Stroke Study has demonstrated that antiplatelet and

anticoagulant treatments are of similar efficacy in preventing recurrent neurologic events in stroke patients with PFO. PFO closure, either surgical or percutaneous, may further reduce event rates; however, this has been demonstratedby the RESPECT trial which compares PFO closure with

medical therapy . For patients with a history of cryptogenic stroke and PFO, the RESPECT Trial provides evidence that closure with the Amplatzer PFO Occluder reduces risk by 46.6 to $72.7 \%$ over medical management alone.
\end{abstract}

Key words: stroke, patent foramen ovale, RESPECT.

Revista Argentina de Cardioangiología Intervencionista 2014;5(2):125-129

\section{INTRODUCCIÓN}

La asociación entre cortocircuitos intracardíacos, stroke criptogénico y migrañas representan un área de investigación que puede tener significativas implicancias para muchos pacientes y puede mejorar nuestra comprensión de dichos fenómenos.

En personas jóvenes, menores de 55 años, la existencia de un stroke de origen isquémico, sin causas embolígenas del lado izquierdo del corazón, debe hacer pensar en la existencia de una embolia paradójica y, por lo tanto, debe descartarse la presencia de un foramen oval permeable. Sin embargo, debemos definir ambas situaciones.

\section{STROKE CRIPTOGÉNICO (SC)}

El diagnóstico de SC es un diagnóstico de exclusión. Ante un stroke de origen isquémico, determinado con

\footnotetext{
. Ex Jefe de Hemodinamia, Hospital Juan P. Garrahan. CABA, Argentina.

2. Cardiólogo infantil, Instituto FLENI. CABA, Argentina.

$\triangle$ Correspondencia: Horacio José Faella. J. B. Alberdi 1137. CABA, Argentina| homefaella@sion.com
}

Conflictos de intereses: no existen
TC o RNM, en personas jóvenes, debe descartarse su origen embólico del lado izquierdo del corazón, esto es: a) de la aurícula izquierda, ante la ausencia de arritmias paroxísticas o permanentes como fibrilación auricular o aleteo, que no haya trombos en la orejuela izquierda, b) del ventrículo izquierdo ante la ausencia de aneurismas y trombos, c) de la aorta ascendente, sin calcificaciones, d) de las arterias de cuello, sin obstrucciones y e) de los vasos intracerebrales. Sólo entonces debe investigarse la presencia de un foramen oval permeable mediante la realización de un eco-Doppler transcraneano o de un ecocardiograma transesofágico con maniobra de Valsalva.

\section{FORAMEN OVAL PERMEABLE (FOP)}

Todos los mamíferos tienen un FOP. Ya que el feto no puede respirar, el oxígeno de la sangre se obtiene a través de la placenta. Los mamíferos tienen un mecanismo para que la sangre oxigenada proveniente de la placenta pase desde la vena cava inferior a través del FOP hacia la aurícula izquierda (AI), luego al ventrículo izquierdo (VI) y a la aorta para finalmente irrigar el cerebro.

El FOP no es un agujero ni un espacio vacío como la comunicación interauricular, sino un pasaje con un flap para permitir el paso entre las aurículas en un solo 
sentido, de derecha a izquierda. Posteriormente al parto, después que los pulmones se expanden, la presión en la AI excede a la de la AD, presionando el septum primum contra el septum secundum y el cortocircuito de derecha a izquierda termina. En la mayoría de los seres humanos, el FO se cierra definitivamente en el primer año de vida. Sin embargo, la ausencia de un tejido de fusión genera un FOP. Dicho foramen permanece permeable en un $25 \%$ de los adultos. ${ }^{2-4}$

A pesar de que el FOP ha sido descripto desde hace muchos años, no se creía que podía tener implicancias clínicas de importancia. Con el advenimiento de la ecocardiografía, se han descripto ejemplos aislados de coágulos a lo largo del FOP cabalgando el septum interauricular. Si estos coágulos, de 1 a $3 \mathrm{~mm}$ de diámetro, pasan a la circulación pulmonar, provocan una pequeña embolia pulmonar que no tiene implicancias clínicas; sin embargo, si ellos pasan a través del foramen y van hacia la circulación cerebral, los efectos son de un impacto devastador.

Deben existir 2 condiciones necesarias para determinar la etiología de un embolismo paradójico. La primera es la presencia de pequeños trombos venosos que pueden estar alojados en el FO mismo, en las venas de la pelvis o en el sistema venoso de los miembros inferiores. Sin embargo, su presencia es difícil de diagnosticar, ya sea con flebografía (sólo el 10\%) o con RNM (20\%). ${ }^{5,6}$ La segunda condición necesaria para una embolia paradójica es un cortocircuito de derecha a izquierda a través del FOP. Utilizando Doppler transcraneano con maniobra de Valsalva o test tusígeno o ecocardiografía transesofágica con dichas maniobras, es posible diagnosticar el cortocircuito en casi el $70 \%$ de los casos en que este existe.

La única forma en que un embolismo paradójico debe considerarse como la etiología de un stroke es cuando una persona joven, menor de 55 o 60 años, sin factores de riesgo obvios predisponentes para stroke, presenta un comienzo súbito de déficit neurológico y un patrón de TC o RMN diagnosticando un accidente isquémico cerebral reciente. ${ }^{7.8}$

\section{EL DIAGNÓSTICO DE STROKE CRIPTOGÉNI- CO ES UN DIAGNÓSTICO DE EXCLUSIÓN}

No debe haber otras anomalías estructurales en la RMN que puedan justificar los hallazgos neurológicos. Tampoco deben existir otras causas de fenómenos embólicos tales como fibrilación auricular, estenosis mitral o aterosclerosis significativa de la aorta ascendente, de los vasos carotídeos o cerebrales.

Como parte de esta evaluación, debe ser rutinario efectuar un ecocardiograma transesofágico con Doppler para evaluar el septum interauricular tanto en reposo con solución salina agitada como con maniobra de Valsalva o test tusígeno.
Una minoría de los pacientes, alrededor del 5\%, manifiesta que su stroke ocurrió con posterioridad a factores predisponentes tales como viajes prolongados en avión o en automóvil. En otro 10\%, los síntomas están asociados a un esfuerzo físico tales como ir al baño o levantar un objeto pesado. Otro factor predisponerte es el embarazo, por la posible presencia de trombosis venosa pélvica.

En todos los pacientes con stroke criptogénico deben descartarse estados de hipercoagulabilidad. La mayor condición prevalente de esta condición es la utilización farmacológica de estrógenos contenidos en las píldoras anticonceptivas o de reemplazo hormonal. El síndrome antifosfolipídico es otro factor a tener en cuenta. ${ }^{7}$

Existen otras condiciones en que puede presentarse el stroke criptogénico atribuible a una embolia paradójica a través de un FOP.

\section{Síndrome neurológico de descompresión en buceadores}

Es debida a la formación de burbujas de nitrógeno en el sistema vascular proveniente de los tejidos en el momento que el buzo asciende a la superficie., ${ }^{9} 10 \mathrm{La}$ incidencia de FOP está aumentada en los pacientes que desarrollan este tipo de enfermedad. Se ve al menos el doble de lesiones isquémicas cerebrales en buzos con FOP que en aquellos que no lo tienen. ${ }^{11,12} \mathrm{El}$ cierre percutáneo del FOP ha sido exitoso en la prevención de la recurrencia de la enfermedad del síndrome neurológico descomprensivo en buzos. ${ }^{13,14}$

\section{Síndrome de platipnea-ortodeoxia}

Es un síndrome raro y pobremente comprendido, en el que los pacientes desarrollan disnea e insaturación arterial en posición erecta. ${ }^{15-17}$ Esta enfermedad se debe a la acentuación ortostática de un cortocircuito de derecha a izquierda a través de una comunicación interauricular, generalmente un FOP. Este síndrome aparece en pacientes con enfermedad pulmonar severa, como, por ejemplo, neumonectomía, embolia pulmonar recurrente o enfermedad pulmonar obstructiva crónica. Las presiones pulmonares son normales. El mecanismo fisiopatológico es desconocido. ${ }^{15} \mathrm{El}$ cierre percutáneo del FOP o la CIA demostró un aumento de la saturación arterial de oxígeno y mejoría de los síntomas respiratorios. ${ }^{17-19}$

\section{Migrañas}

A pesar de los avances médicos en el tratamiento de las migrañas, muchos pacientes siguen sufriendo episodios frecuentes e inhabilitantes. ${ }^{20}$

El 12\% de la población (18\% de mujeres y 6\% de hombres) está afectado por migrañas. Las migrañas constituyen, además, un factor de riesgo para la aparición de stroke en pacientes jóvenes, especialmente en mujeres que ingieren anticonceptivos orales. ${ }^{21}$ 


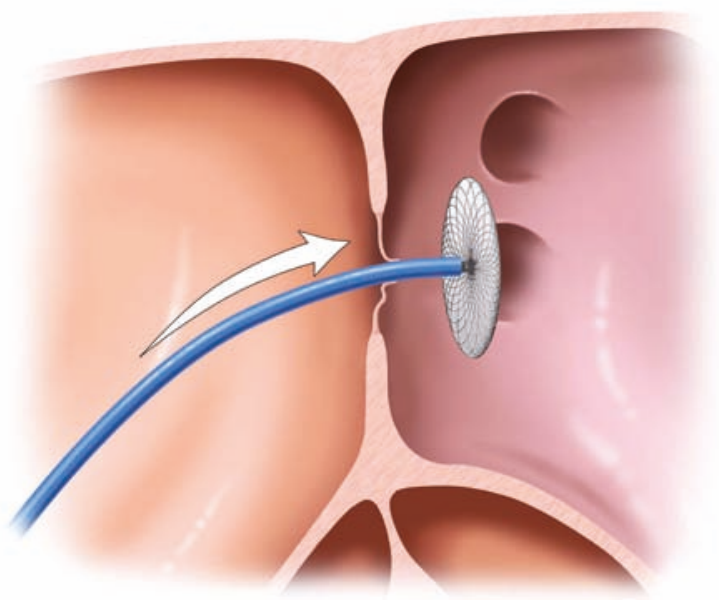

Figura 1. Se observa la camisa que pasa a través del FOP y la guía liberadora que empuja y abre el disco distal en la aurícula izquierda.

El estudio epidemiológico más importante sobre la relación entre stroke criptogénico, FOP y migrañas fue publicado en 2002 por Lamy et al. ${ }^{22}$ En 500 pacientes con stroke criptogénico, 46\% tenían un FOP, y la migraña era 2 veces más común entre los pacientes con FOP (28\%) que en aquellos que no lo tenían (14\%). Diversos estudios sugieren que el cortocircuito de derecha a izquierda con microembolias puede jugar un rol importante en la etiología de la migraña..$^{23,24}$ Otra hipótesis es que el FOP permite el pasaje de derecha a izquierda de sustancias químicas que podrían actuar como gatillo para el desarrollo de la migraña en pacientes susceptibles. Si no tuvieran FOP, estas sustancias pasarían primero a través de los pulmones donde podrían degradarse, diluirse o metabolizarse..$^{25,26}$

Diversos autores han descripto una disminución o desaparición de las migrañas en pacientes a los que se les cerró en forma percutánea el FOP o la CIA..$^{27,28}$

\section{INDICACIONES DEL CIERRE PERCUTÁNEO DEL FOP}

\section{Absolutas}

1. Stroke criptogénico recurrente a pesar del tratamiento con antiagregantes o anticoagulantes.

2. Stroke criptogénico en pacientes con contraindicación para el tratamiento con antiagregantes o anticoagulantes.

3. Sindrome de platipnea-ortodeoxia.

\section{Relativas}

1. Buceadores.

2. Pacientes con migrañas inhabilitantes.

3. Embolismo pulmonar + FOP.

4. Estados de hipercoagulabilidad.

5. Primer stroke criptogénico y FOP.

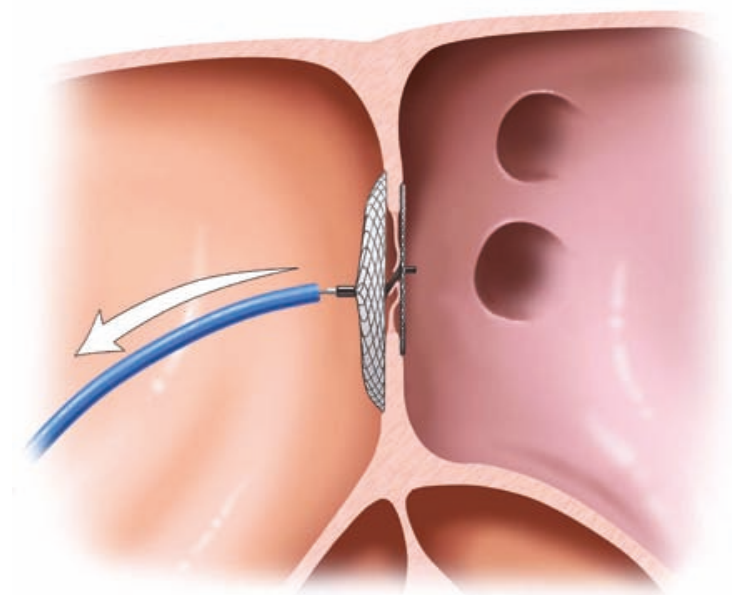

Figura 2. Al retraer todo el sistema, el disco distal se apoya contra el tabique interauricular, y retirando la camisa en el sentido de la flecha se abre el disco proximal en la auricula derecha.

Son necesarios estudios clínicos multicéntricos, aleatorizados, prospectivos que comparen el tratamiento médico con el cierre percutáneo del FOP para que este se convierta en una indicación absoluta.

\section{NUESTRA EXPERIENCIA CON EL CIERRE PERCUTÁNEO DEL FOP}

Hemos realizado, entre octubre 2002 y marzo 2014, el procedimiento del cierre del FOP por cateterismo en 82 pacientes consecutivos con edades entre 25 y 70 años (mediana 42). El mecanismo de colocación se puede observar en las Figuras 1 a 3.

Setenta y siete ( 44 mujeres y 33 hombres) habían padecido stroke criptogénico, 2 eran buzos y 3 sufrían migrañas.

El diagnóstico de FOP se realizó con ecocardiograma transesofágico con inyección de burbujas con prueba de Valsalva y se controló de esa manera durante todo el procedimiento, durante los 2 últimos años; el control se efectuó con ecocardiograma intracardíaco.

Realizamos punción percutánea de la vena femoral derecha en todos los pacientes. Con catéter de orificio terminal progresamos hasta la vena pulmonar izquierda superior a través del FOP. Con guía de intercambio en J retiramos catéter y entonces colocamos introductor y camisa para punción transeptal de Mullins de 9 French. Se retiró entonces el introductor dejando la camisa y a través de ella, con guía liberadora, se progresó oclusor $\mathrm{PFO}$ Amplatzer de 25 o $35 \mathrm{~mm}$ de diámetro NR (SJM).

Una vez que se exteriorizó el disco distal en la aurícula izquierda (Figura 1), se retrajo todo el sistema de manera de apoyar el disco contra el tabique interauricular y manteniendo firme la guía liberadora se retiró la camisa; de esa manera se abrió el disco proximal en la 


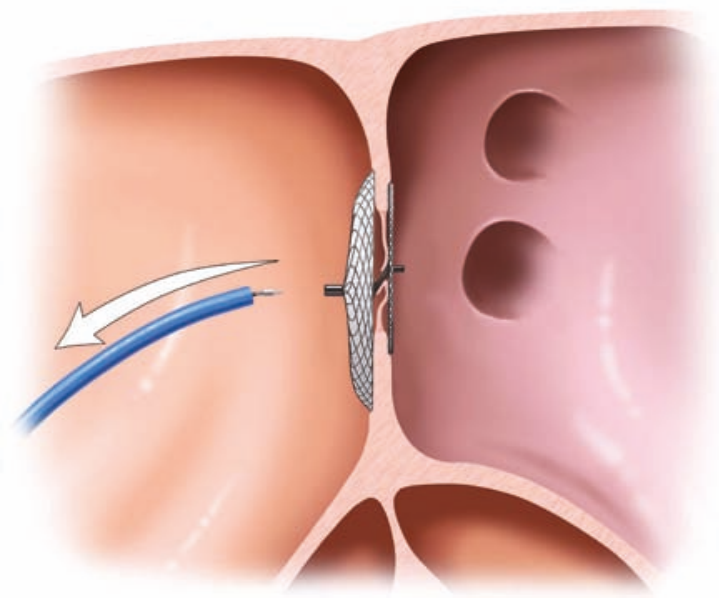

Figura 3. Una vez comprobada la posición del oclusor, rotando la guía liberadora en el sentido contrario a las agujas del reloj, se libera el dispositivo, que ocluye el FOP.

auricula derecha (Figura 2). Se comprobó su correcta posición por el ecocardiograma y tirando y empujando suavemente de la guía liberadora. La liberación del oclusor (Figura 3) se logró rotando en forma antihoraria la guía liberadora.

Todos los pacientes fueron medicados posteriormente con aspirina $80 \mathrm{mg} /$ día durante 6 meses y clopidogrel $75 \mathrm{mg} /$ día por 3 meses.

En nuestra serie no hubo complicaciones relacionadas con el método, como embolizaciones, ni erosiones. Se produjo cierre completo en todos los pacientes a los 6 meses con control por ecocardiograma transtorácico.

No existió stroke recidivante en ningún paciente.

Estos resultados están de acuerdo con los reportados por el RESPECT trial, en el cual se demostró que, en pacientes con historia de stroke criptogénico y FOP, el cierre del FOP con el oclusor Amplatzer redujo el riesgo de recurrencia entre 46,6 y el $72,7 \%$ en comparación con el tratamiento médico, aunque los resultados no fueron estadísticamente significativos por el tamaño de la muestra. ${ }^{29}$

\section{BIBLIOGRAFÍA}

1. Overell JR, Bone I, Lees KR. Interatrial septal abnormalities and stroke: a meta analysis of case control studies. Neurology 2000;55(8):1172-1179.

2. Wahl A, Windecker S, Meier B. Patent foramen ovale: pathophysiology and therapeutic options in symptomatic patients. Minerva Cardioangiol 2001;49(6):403-411.

3. Hagen PT, Scholz DG, Edwards WD. Incidence and size of patent foramen ovale durins the first decades of life: an autopsy study of 965 normal hearts. Mayo Clin Proc 1984;59(1):17-20.

4. KerutE, Norfleet W, Plotnick G, Giles T.Patentforamen ovale: a review of associated conditions and the impact of physiological size. JACC 2001;38(3):613-623.

5. Lethen H, Flachskampf FA, Schneider R, et al. Frequency of deep vein thrombosis in patients with foramen ovale and ischemic stroke or transient ischemic attack. Am J Cardiol 1997;80(8):1066-1069.

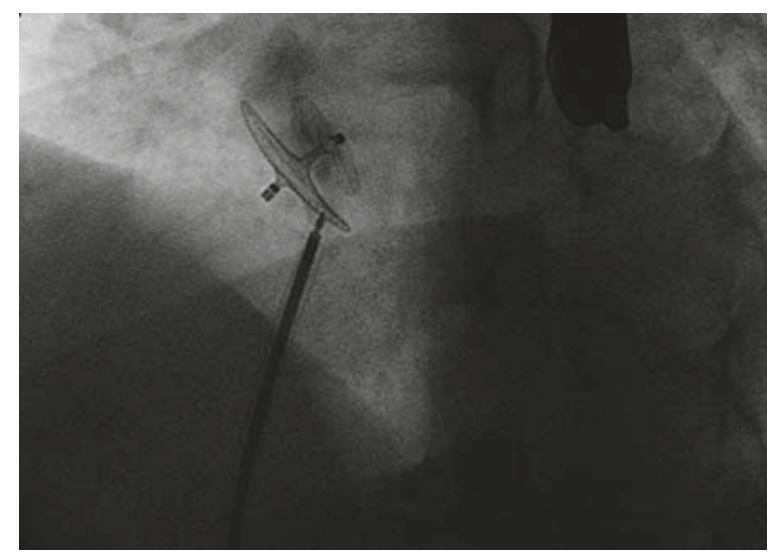

Figura 4. Se observa el dispositivo ya liberado. Se ve, además, la sonda del eco transesofágico

\section{CONCLUSIONES}

1. El cierre del FOP en pacientes menores de 55 años con stroke criptogénico es un tratamiento alternativo al tradicional (antiagregantes o anticoagulantes) y a la cirugía.

2. Son necesarios estudios aleatorizados que demuestren que, en estos casos, el cierre por dispositivo es una indicación absoluta.

3. Stroke criptogénico o ACVI recurrente $+\mathrm{FOP}=$ cierre percutáneo del FOP.

4. Stroke criptogénico o ACVI + FOP + aneurisma del septum interauricular $(4 \%)=$ cierre percutáneo.

5. Stroke criptogénico o ACVI + FOP + trombosis venosa profunda $=$ cierre percutáneo.

6. Stroke criptogénico o ACVI + FOP + antecedentes de $S C$ = cierre percutáneo.

\section{AGRADECIMIENTOS}

Los autores agradecen a los Dres. Luis De La Fuente y a Jorge Mrad como responsables de los Servicios de Hemodinamia de los Sanatorios Suizo, Los Arcos y Instituto Argentino de Diagnóstico y Tratamiento por haber confiado en ellos para colaborar en la resolución de sus pacientes.
6. CramerSC, Rordorf G, Maki JH, et al. Increased pelvis vein thrombi in cryptogenic stroke: results of the Paradoxical Embolifrom Large Veins in Ischemic Stroke (PELVIS) study. Stroke 2004;35(1)46-50.

7. Kedia G, Tobis J, Lee MS B. Patent foramen ovale: clinical manifestations and treatment. Rev Cardiovasc Med 2008;9(3):168-173.

8. Lechat P, Mas JL, Lascault $G$, et al. Prevalence of patent foramen ovale in patients with stroke. N Engl J Med 1988;318(18):1148-1152.

9. Barrat DM, Harch PG, Van Meter K. Decompression illness in divers: a review of the literature. Neurologist 2002;8(3):186-202.

10. GermonpreP, DendaleP, Unger P, Balestra C. Patent foramen ovale and decompression sickness in sports divers. J Appl Physiol 1998;84(5):1622-1626.

11. Moon RE, Camporesi EM, Kisslo JA. Patent foramen ovale and decompression sickness in divers. Lancet 1989;1(8637):513-514. 
12. Schwerzmann M, Seiler C, Lipp E, et al. Relation between directly detec ted patent foramen ovale and ischemic brain lesions in sport divers. Ann Intern Med 2001;134(1):21-24.

13. Knauth M, Ries S, Pohimann Set al. Cohort study of multiple brain lesions in sport divers: role of a patent foramen ovale. BMJ 1997;314(7082):701-705.

14. Walsh KP, Wilmshurst PT, Morrison WL. Transcatheter closure of patent foramen ovale using the Amplatzer septal occluder to prevent recurrence of neurological decompression illness in divers. Heart 1999;81(3):257-261.

15. Seward JB, Hayes DL, Smith HC, et al. Platynea-orthodeoxia: clinical profile, diagnostic workup, management, and report of seven cases. Mayo Clin Proc 1984:59(4):221-231.

16. Sorrentino M, Resnekov L. Patent foramen ovale associated with platynea and orthodeoxia. Chest 1991;100(4):1157-1158.

17. Rao PS, Palacios IF, Bach RG, Bitar SR, Sideris EB. Platynea-orthodeoxia: management by transcatheter buttoned device implantation. Catheter Cardiovasc Interv 2001;54(1):77-82.

18. Guerin P, Lambert V, Godart F, et al. Transcatheter closure of patent foramen ovale in patients with platypnea-orthodeoxia: results of a multicentric French registry. Cardiovasc Intervent Radiol 2005;28(2):164-168.

19. Delgado G, Inglessis I, Martin-Herrero F, et al. Management of platypnea-orthodeoxia syndrome by transcatheter closure of atrial communication: hemodynamic characteristics, clinical and echocardiographic outcome. J Invasive Cardiol 2004,16(10)578-582.

20. Mathew NT. Pathophysiology, epidemiology, andimpact of migraine. Clin Cornerstone 2001:4(3):1-17.
21. Lipton RB, Scher Al, Kolodner K, Liberman J, Steiner TJ, Stewart WF. Migrainein the United States: epidemiology and patterns of health care use. Neurology 2002;58(6):885-894.

22. Lamy C, Giannesini C, Zuber N, et al. Clinical and imaging findings in cryptogenic stroke patients with and without patent foramen ovale: the PFOASA Study. Atrial Septal Aneurysm. Stroke 2002;33(3):706-711.

23. Lechner H, Ott E, Fazekas F, Pilger E. Evidence of enhanced platelet aggregation and platelet sensivity in migraine patients. Cephalalgia 1985:5(2):89-91.

24. Fragoso YD. Reduction of migraine attacks during the use of warfarin Headache 1997;37(10):667-668.

25. Sarchielli P, Alberti A, Vaianella L, et al. Chemokine levels in the jugularvenous blood of migraine without aura patients during attacks. Headache 2004:44(10):961-968

26. SarchielliP, GallaiV.Nervegrowthfactor and chronic daily headache:apotential implication for therapy. Expert Rev Neurother 2004;4(1):115-127.

27. Azarbal B, Tobis J, Suh W, Chan V, Dao C, Gaster R. Association of interatrial shunts and migraine headaches: impact of transcatheter closure. JACC 2005;45(4):489-492.

28. Yew W, Wilson NJ. Transcatheter atrial septal defect closure with the Amplatzer septal occluder: five-year follow-up. Catheter Cardiovasc Interv 2005;64(2):193-196

29. Carroll JD, Saver JL, Thaler DE, et al. Closure of patent foramen ovale versus medical therapy after cryptogenic stroke. NEngl J Med 2013;368:10921100 . 